\title{
Genetic Polymorphisms Association in Restenosis of Coronary Arteries
}

Dana Taizhanova ${ }^{1}$, Roza Bodaubay ${ }^{2}$, Aliya Toleuova ${ }^{1}$, Akerke Kalimbetova $^{2 *}$, Dmitriy Babenko ${ }^{2}$, Anar Turmukhambetova ${ }^{2}$, Ludmila Akhmaltdinova ${ }^{2}$, Olga Visternichan ${ }^{1}$

${ }^{1}$ Department of Internal Diseases, NCJSC "Medical University of Karaganda," Karaganda, Kazakhstan; ${ }^{2}$ Scientific and Research Center (SRC), NCJSC "Medical University of Karaganda”, Karaganda, Kazakhstan

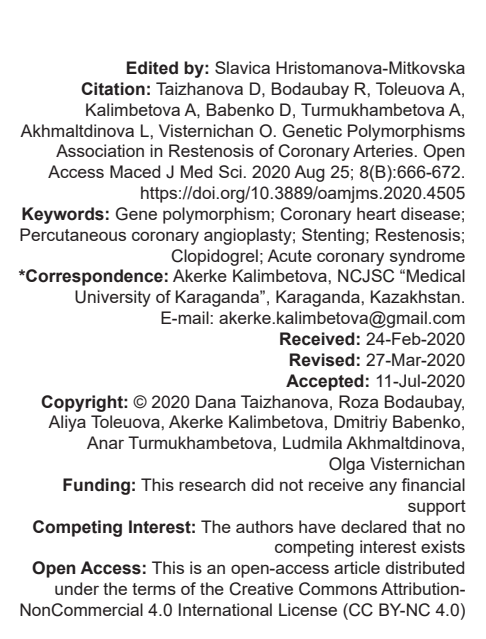

Abstract

BACKGROUND: There is a reason to believe that the polymorphism of genes encoding some enzymes and receptors plays a role in increasing of restenosis development risk. It is common knowledge that ethnicity affects the frequency of heterozygous genotypes occurrence. There is the evidence that polymorphism of the FGB gene (rs1800790) and THBD gene was determined in the ethnic group of Kazakhs with restenosis of the coronary arteries, which can be considered as genetic predictors of restenosis development. Today, the questions of the role of the genetic component in the development of coronary heart disease (CHD) remain open.

AIM: Evaluation of gene polymorphism in patients with restenosis of coronary arteries after stent installation.

MATERIALS AND METHODS: The group consisted of Kazakh population of the age category from 45 to 65 years of both sexes: Group I (50 persons) patients with a diagnosis of CHD, with a fixed stent and the development of restenosis during the year; Group 2 (58 persons) - with a fixed stent and no restenosis during the year. The association of genetic polymorphisms was evaluated in accordance with the case-control design based on the generalized linear model assuming a log-additive inheritance model.

RESULTS: Thus, when comparing two groups using five patterns of inheritance, the following SNP were revealed: Codominant inheritance pattern - rs1045642 ( $p=0.0427)$, dominant inheritance pattern $-r s 12041331(p=0.036088)$ rs13431554 ( $p=0.025461)$, and $r s 1045642(p=0.012774)$, and overdominant inheritance pattern - rs12041331 $(p=0.051736)$, rs5918 ( $p=0.057652)$, and rs13431554 ( $p=0.036006)$. Thus, three SNPs associated with stenting were identified: rs7543130 ( $p=0.009324)$, rs6785930 ( $p=0.016858)$, and rs7819412 ( $=0.061325)$ and two SNPs associated with the development of restenosis after stent placement: rs1061781 $(p=0.063184)$ and $r s 342293(p=0.061636)$.

CONCLUSION: The polymorphisms associated with the risk of developing restenosis after stenting were determined: Codominant inheritance pattern - one polymorphism (rs1045642, $p=0.0427$ ); dominant inheritance pattern - three polymorphisms ( $r$ 12041331, $p=0.036088 ; r s 13431554, p=0.025461 ; r s 1045642, p=0.012774$ ), and overdominant inheritance pattern - one polymorphism (rs13431554, $\mathrm{p}=0.036006)$. Based on the hybrid machine learning approach (RuleFit), four rules were obtained for assessing the empirical risk of restenosis developing after stenting - from $20 \%$ to $40 \%$.

\section{Introduction}

The significance of personalized medicine development determines the necessity of individual approach to the treatment and prevention of heart diseases and requires accounting molecular and biological differences, and above all the genetic conditionality of pathology.

There is evidence of the influence of various factors on the risk of coronary artery restenosis development: From the intracoronary intervention, the age of the patient, the concomitant diseases, the degree and extent of the lesion, the length of the lesion, the diameter of the vessel lumen after implantation, and etc., to the resistance to pharmacotherapy.

Personalized medicine is a promising direction in the medical science development, aimed at the assessing of the specific biomarkers of the disease, as well as the genetic profile of the patient, which is a key point in the increasing of the treatment effectiveness.

Endovascular treatment methods, such as stenting of coronary arteries, are widespread in the treatment of coronary heart disease (CHD), but restenosis in the stent is the main limitation of this method effectiveness, and even the use of drug-coated stents has not completely resolved the problem [1].

Stenting of the coronary arteries remains an effective method at coronary arteries revascularization, in this regard, the search for new predictors of restenosis development after stent fixing is relevant.

There is a reason to believe that the polymorphism of genes encoding some enzymes and receptors plays a role in increasing of restenosis development risk [2]. A number of large-scale programs 
(aimed at studying of restenosis genetics) are currently known, such as genetic determinants of restenosis, Coronary AngioPlasty Amlodipine REStenosis Study, research, and ISAR-strut thickness effect on restenosis outcome 2 [3], [4]. The development of restenosis in the stent can also indirectly depend on polymorphisms in the genes of the hemostatic system, renin-angiotensin system, and antioxidant system [5].

As it is known that L54M (rs854560) and Q192R (rs662) polymorphisms have the greatest clinical significance among all the polymorphic variants of the PON1 gene [6], [7], [8], [9]. The CYP2C19 gene has nine exons and it is highly polymorphic with more than 25 allele variants [5]. Using an algorithm in the R statistics environment of the SNPedia base, it was performed an analysis for the presence of PON1, CYP2C19 gene variations as predictors of the risk of development of coronary artery disease and coronary artery restenosis. The distribution data were obtained in various populations of the following alleles - L54M (rs854560) and Q192R (rs662) rs12248560, rs41291556, rs41291556, rs72552267, rs4986893, rs4244285, rs72558186 (A) represents SNP CYP2C19, and rs56337013.

It is common knowledge that ethnicity affects the frequency of heterozygous genotypes occurrence. Thus, there is the evidence that the frequency of the occurrence of heterozygous genotypes of CYP1C19*2GA $\left({ }^{*} 1 /{ }^{*} 2\right)$ alleles ranges from $28 \%$ to $60 \%$ in the Asian population, according to various authors [5], [9]. As a result of scientific research, there was a difference in the prevalence of polymorphism of the CYP2C19 isoenzyme in different ethnic groups. The frequency of the occurrence of this allele is about $12 \%$ in Europeans, $12 \%$ in African-Americans, and $29-35 \%$ in Asians [5]. According to other sources [9], the prevalence of the allele with the CYP2C19*2 genotype is about 25-30\% in Europeans and 50-60\% in Asians [5].

A decrease in the effectiveness of clopidogrel antiplatelet agent in different patients depending on ethnic groups is associated with the genetic polymorphism of CYP2C19, which also increases the risk of coronary artery restenosis.

There is the evidence that polymorphism of the FGB gene (rs1800790) and THBD gene was determined in the ethnic group of Kazakhs with restenosis of the coronary arteries, which can be considered as genetic predictors of restenosis development [10].

It was demonstrated that the distribution frequency of the *2 allele (GA and $A A$ ) for the G681A polymorphic marker CYP2C19 gene in patients of Kazakh nationality with acute coronary syndrome was $27 \%$ and $2 \%$, respectively, while the distribution frequency for the allele *3 (GA and AA) amounted to $9 \%$ and $1 \%$, respectively [11].

Today, the questions of the role of the genetic component in the development of $\mathrm{CHD}$ remain open, in particular, at the risk of repeated stenosis developing in the area of the stent installation, as well as in determining its significance in the structure of risk factors and causes in cases of low effectiveness of therapy, which is generally aimed at optimizing of a personalized approach in the treatment and tactics of monitoring the patients with coronary artery disease.

\section{Methods}

The studies were conducted on the base of Karaganda Medical University, clinics of Karaganda. Permission of the Committee on Bioethics of KSMU No.305 dated May 19, 2017, was obtained for research conduction.

The persons of Kazakh ethic nationality were included to the study. Group I consisted of 50 persons of the age category from 45 to 65 years of both sexes. Inclusion criteria: Patients with a diagnosis of $\mathrm{CHD}$, with a fixed stent and the development of restenosis during the year (according to coronary angiography and medical records) based on the recommendation of the European Society of Cardiology (ESC). Group 2 consisted of 58 persons at the age from 45 to 65 years of both sexes. Inclusion criteria: Patients with a diagnosis of coronary artery disease, with a fixed stent and no restenosis during the year (according to the medical record) based on the recommendation of the ESC.

Exclusion criteria for Groups 1 and 2: Chronic heart failure in the stage of decompensation (Stage II B according to the classification of Strazhesko-Vasilenko and FC IV according to NYHA); life-threatening heart rhythm disturbances; chronic rheumatic heart disease; acute cerebrovascular accident; bronchial asthma; chronic obstructive pulmonary disease of 2-3 stage; decompensated liver cirrhosis; severe renal impairment (glomerular filtration rate $<30 \mathrm{ml} / \mathrm{min}$ ); malignant neoplasms; morbid obesity; and the presence of gout.

The association of genetic polymorphisms was evaluated in accordance with the case-control design based on the generalized linear model assuming a logadditive inheritance model.

Statistical analysis was carried out in the programs of $\mathrm{R}$ statistics (Compare Groups $\mathrm{R}$ packages http://www.jstatsoft.org/ [10]), Statistica 6.0 (Stat-Soft), and SPSS (IBM) under the following conditions:

- Quantitative data with normal distribution: Student's t-test for two groups or analysis of variance, when the number of groups is more than 2;

- Quantitative data with abnormal distribution: Non-parametric Mann-Whitney and KruskalWallis criteria for independent groups and Wilcoxon's criterion for dependent groups; 
Categorical data: Chi-square criterion or Fisher's exact test if necessary (when the expected frequency is $<5$ in one of the cells).

Assessment of the data distribution (normality test) was carried out on the basis of the Shapiro-Wilks criterion. $p<0.05$ was taken for the level of statistical significance of differences in indicators. The data in the text and tables are presented as the median (Me), quartile 1, and quartile $3(\mathrm{Q} 1, \mathrm{Q} 3)$.

The study of the markers diagnostic value was carried out using receiver operating characteristic curves, which reflect the specificity and sensitivity of the test. The quality of the model with the area under the curve (AUC): $0.9-1.0$ is considered to be excellent, 0.9-0.8 - very good, and 0.7-0.8 - good.

Search and assessment of risk factors associated with the phenotype (disease) were carried out using RuleFit - a hybrid algorithm for regression and classification with increasing gradient and LASSO algorithm for training a highly accurate and interpretable model (Figure 1) [11].

\section{Results}

To identify statistically significant genetic polymorphisms with the development of restenosis, two groups were selected: Patients with stenting without restenosis (this group was considered as a control) and patients with the development of restenosis in the course of the past year.

As the number of patients in each group is relatively small, five different inheritance models were used to identify the genotype-phenotype association: Dominant, codominant, recessive, overdominant, and log-additive models of inheritance. The results of the analysis are presented in Figure 2 and Table 1.

Table 1: Table of genetic polymorphisms associated with restenosis after stent fixation

\begin{tabular}{llll}
\hline Polymorphism & Inheritance models & $\mathrm{p}$ & Comment \\
\hline rs1045642 & Codominant & 0.0427 & - \\
rs12041331 & Dominant & 0.036088 & - \\
rs13431554 & Dominant & 0.025461 & - \\
rs1045642 & Dominant & 0.012774 & - \\
rs12041331 & Overdominant & 0.051736 & Close to threshold value \\
rs5918 & Overdominant & 0.057652 & Close to threshold value \\
rs13431554 & Overdominant & 0.036006 & - \\
\hline
\end{tabular}

The analysis revealed a total of four different polymorphisms that are associated with the development of restenosis. Assuming a codominant inheritance model, one polymorphism was identified, assuming a dominant and overdominant inheritance model - three SNPs each. In the recessive and logadditive model of inheritance, statistically significant polymorphisms associated with the development of restenosis were not obtained.

Two machine learning methods were used to assess the associations of polymorphisms and the development of restenosis: Additive Tree [11], [12] and RuleFit [13].

All missing values (positions in which genotypes were not determined for certain reasons) were filled using the Random Forest algorithm (RF = Random forest).

As a result of training based on genotypes associated with the risk of developing restenosis, it was obtained a model with indicators of $100 \%$ specificity and $89 \%$ sensitivity (Figure 3 ). The resulting trained model based on the additive tree algorithm had $94 \%$ balanced accuracy of the predicted results.

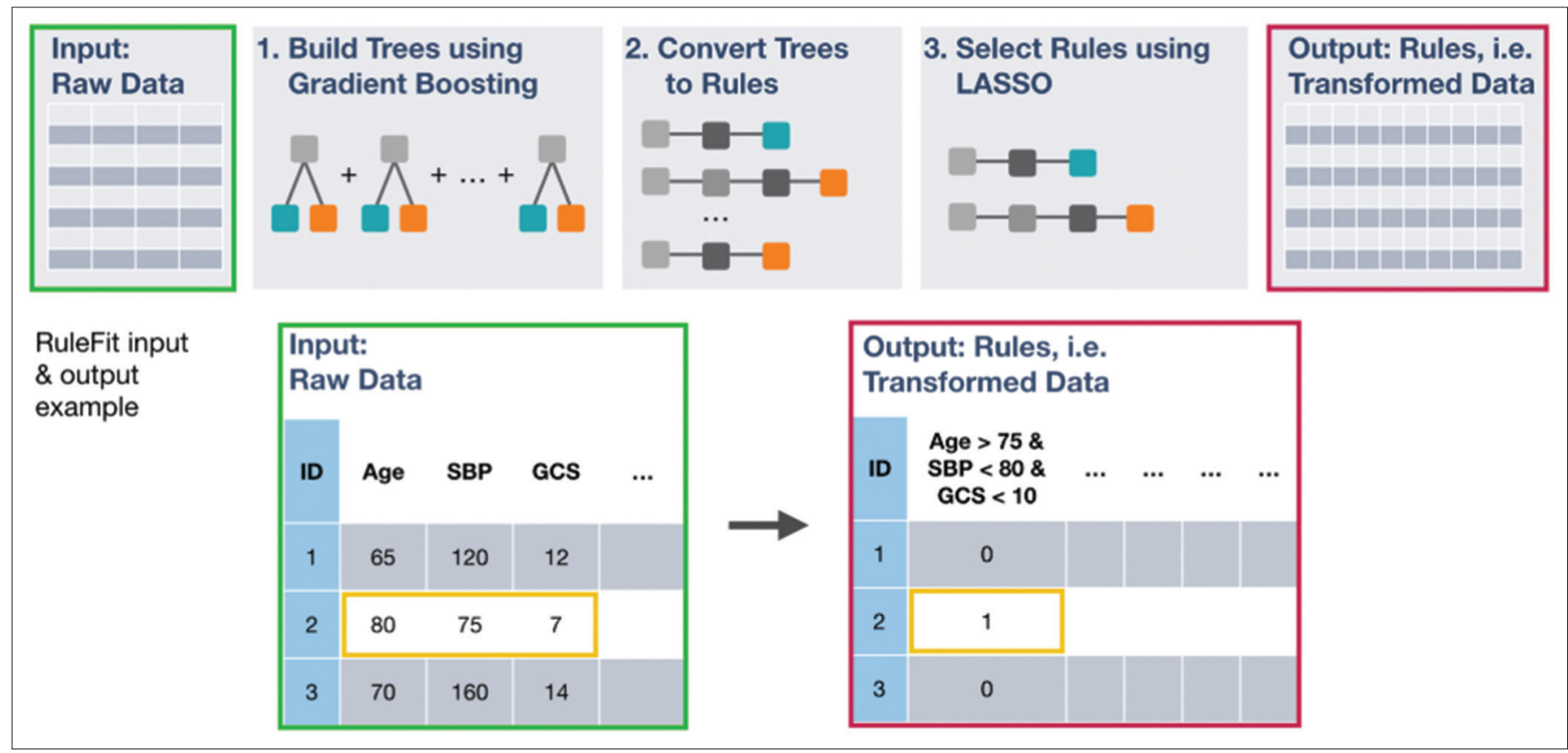

Figure 1: Schematic representation of the analysis stages based on the RuleFit hybrid algorithm 


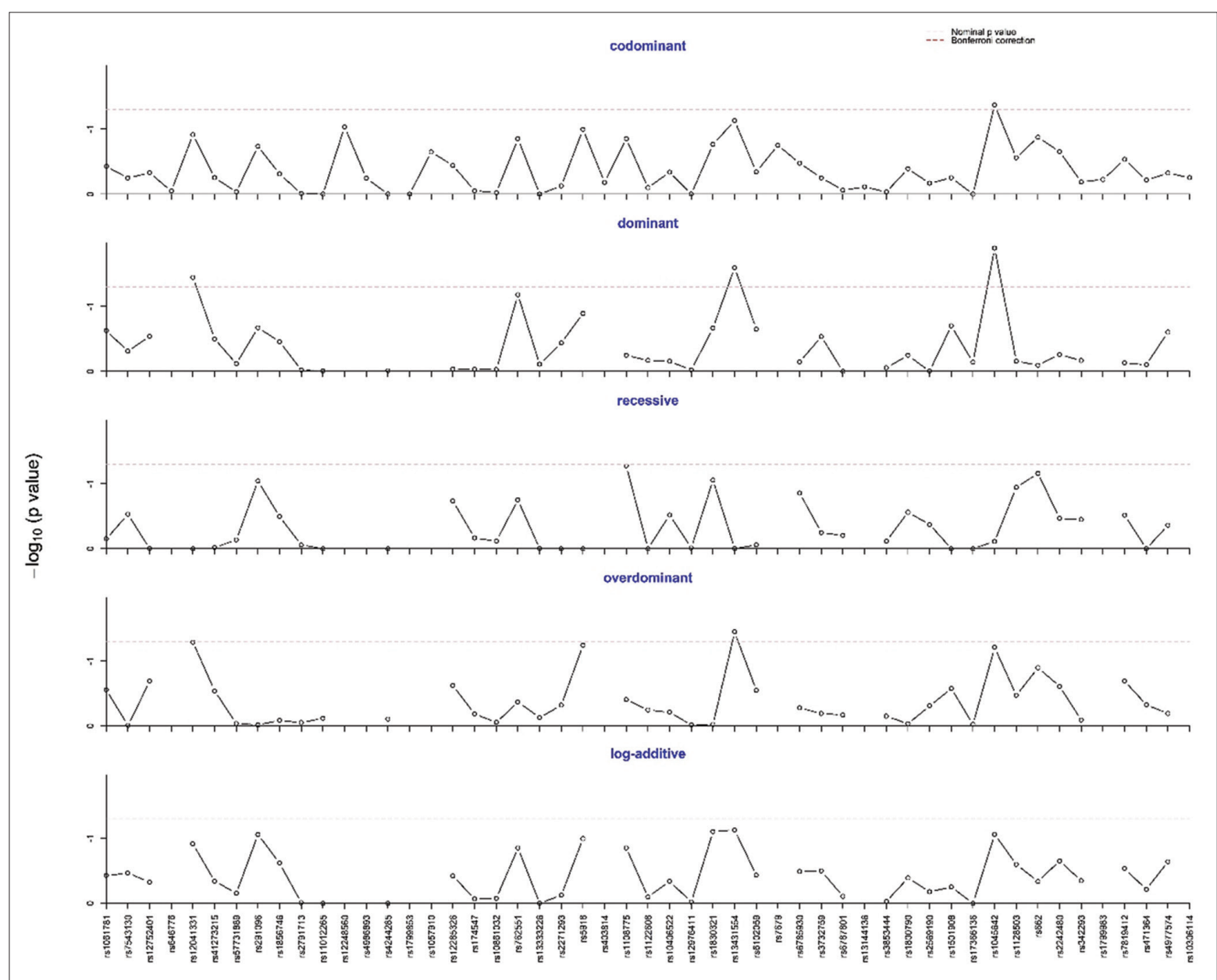

Figure 2: Graphic diagram of p-values obtained when calculating the genotype-restenosis association after stent fixation

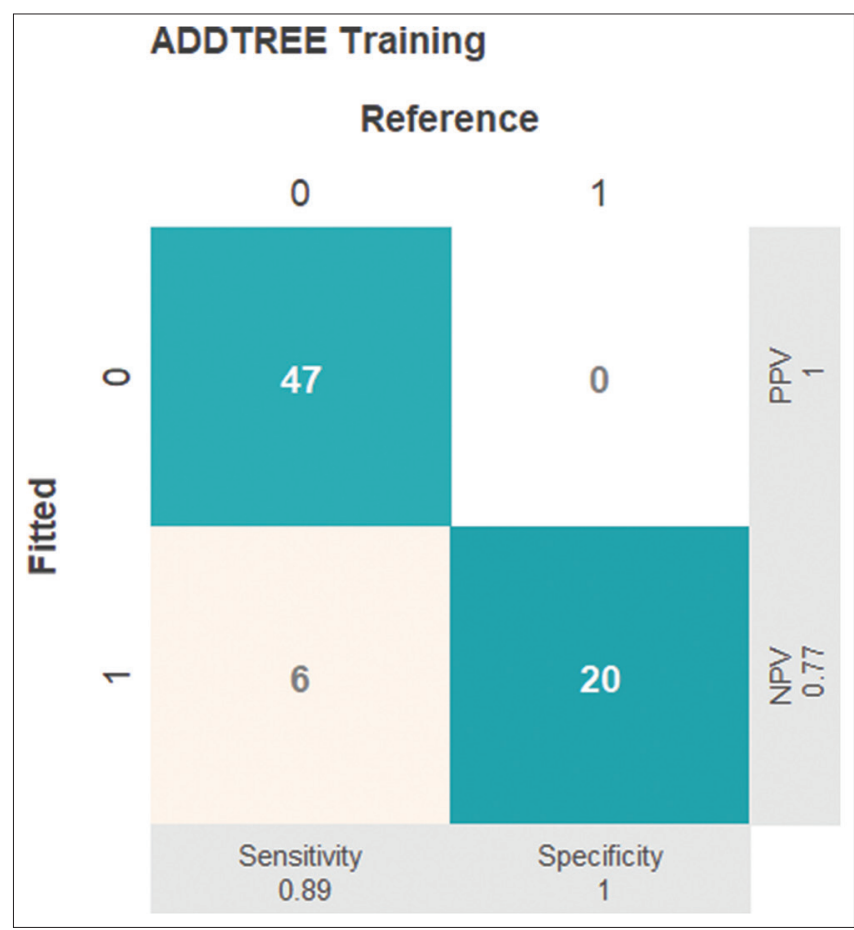

Figure 3: Indicators of a trained model based on additive tree (genotype-restenosis)

Based on the trained model, a decision tree was obtained - an algorithm for analyzing the genotype-restenosis association (Figure 4). As shown in this diagram, the total number of polymorphisms that predict the development of restenosis is nine SNPs. The starting position is the determination of the genotypes of rs1045642 polymorphism.

The machine learning model (RuleFit) after learning achieved the following indicators: Sensitivity $91 \%$, specificity $75 \%$, and AUC $90 \%$ (Figure 5 ). This model had a balanced forecast accuracy of $83 \%$.

This model identified four rules (genotypes of certain polymorphisms) among 519 rules for the assessment of the empirical risk of restenosis developing (Table 2).

Table 2: Table of rules for associations of polymorphism genotypes with the empirical risk of restenosis

\begin{tabular}{|c|c|c|}
\hline Rule & Cases & Empirical risk of restenosis \\
\hline rs $1045642<=2.5+$ rs12041331>2.5 + & 20 & 0.4 \\
\hline $\begin{array}{l}\text { rs } 1501908<=1.5 \\
\text { rs } 1045642<=2.5+\text { rs } 13431554<=1.5+ \\
\text { rs } 6102059>1.5+\text { rs } 174547>1.5\end{array}$ & 14 & 0.286 \\
\hline rs $1045642<=2.5+$ rs $12041331>2.5+$ rs $662<=2.5$ & 13 & 0.231 \\
\hline $\begin{array}{l}+ \text { rs } 4977574>1.5 \\
\text { rs } 762551<=1.5+\text { rs } 13431554<=1.5+ \\
\text { rs291096>1.5 + rs } 12248560<=1.5\end{array}$ & 10 & 0.2 \\
\hline
\end{tabular}

It should be noted that these machine learning methods work with quantitative data and it required the encoding of genotypes into integer values. 


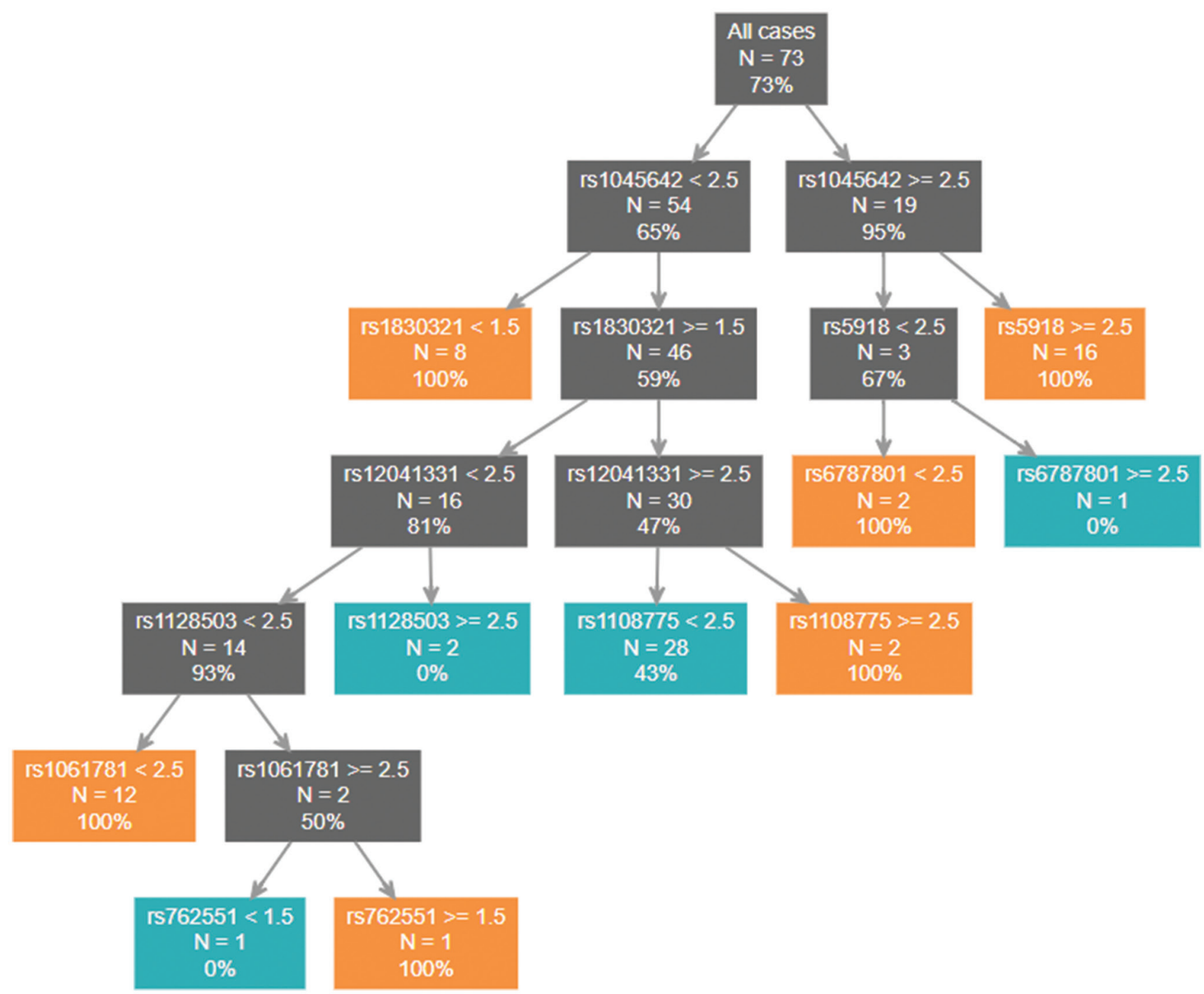

Figure 4: The decision tree algorithm for evaluating the genotype-restenosis association (orange - restenosis after stenting, turquoise stenting without restenosis (control), gray-intermediate state that requires the following definition of polymorphism)

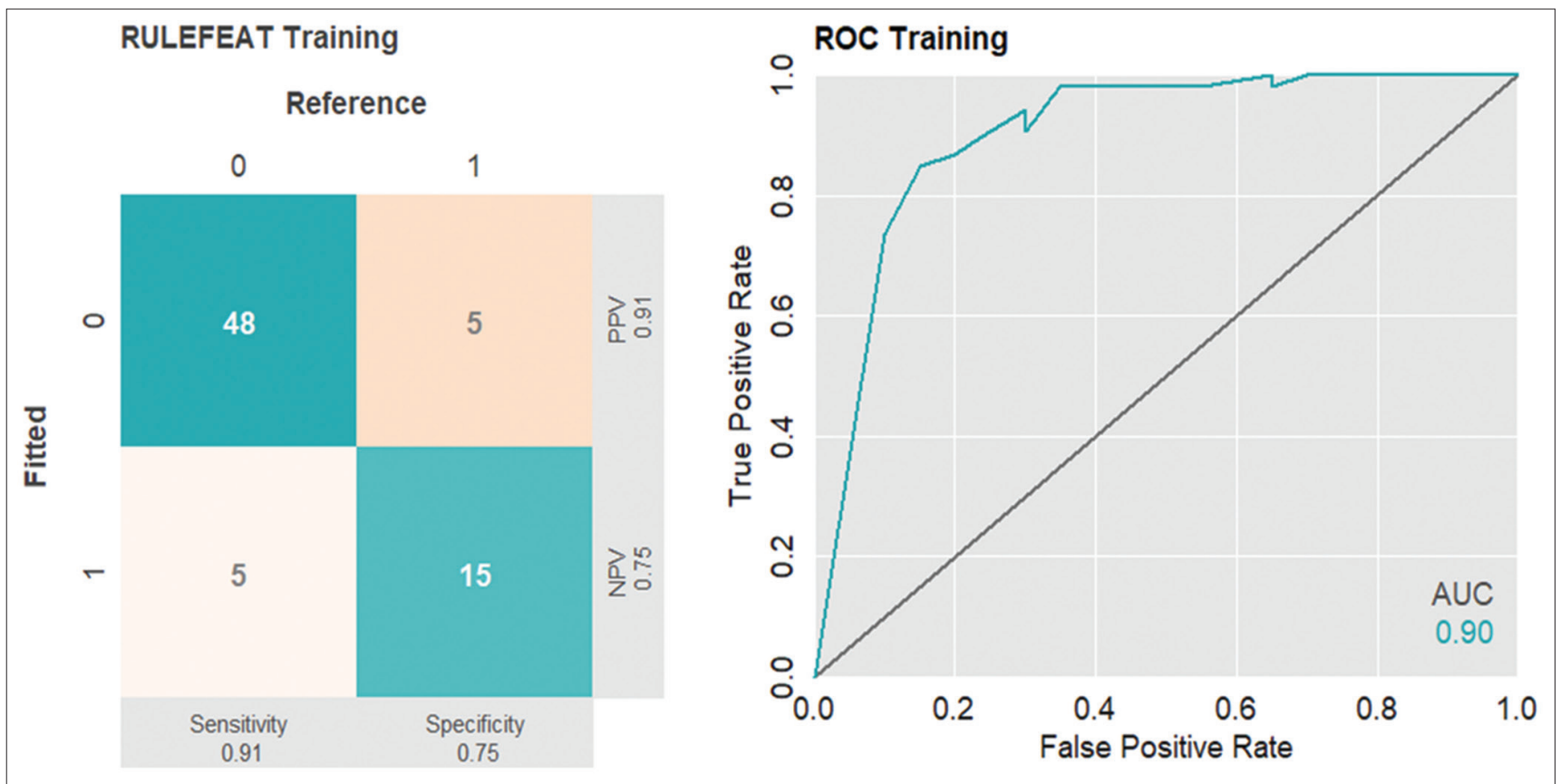

Figure 5: Indicators of a trained model based on RuleFit (empirical risk of restenosis developing after stenting) 
Thus, when comparing two groups (patients without stent restenosis and patients with stent and restenosis) using five patterns of inheritance, the following SNP was revealed: Codominant inheritance pattern - rs1045642 $(p=0.0427)$, dominant inheritance pattern - rs12041331 ( $p=0.036088)$, rs13431554 $(p=0.025461)$, and $r s 1045642(p=0.012774)$, and overdominant inheritance pattern - rs12041331 $(p=0.051736)$, $r s 5918(p=0.057652)$, and $r s 13431554$ $(p=0.036006)$.

The model, trained on the results of genotyping in patients with the development of restenosis after stent fixing using the additive tree algorithm, possessing high specificity (100\%), sensitivity (89\%), and $94 \%$ balanced prediction accuracy, allowed us to create a diagnostic association assessment algorithm polymorphisms with a risk of developing restenosis after stenting in the decision tree form. The decision tree is based on the determination of the rs 1045642 genotype with a pharmacogenetic profile, including the risk of restenosis developing in the treatment by clopidogrel.

Machine learning based on the RuleFit algorithm with $91 \%$ sensitivity, $75 \%$ specificity, and $83 \%$ prognostic accuracy allowed to define a list of four rules for assessing the relationship of the totality of genotypes of certain polymorphisms with the empirical risk of restenosis developing after stenting.

The following gene combinations were identified during the detection of polymorphisms associated with restenosis against the background of antiplatelet therapy with clopidogrel: rs $1045642<=2.5$ + rs12041331>2.5 + rs1501908<=1.5 (revealed in 20 of 50 cases $(40 \%)$. The combination of genes rs $1045642<=2.5+$ rs $13431554<=1.5+$ rs6102059>1.5 + rs174547>1.5 - was identified in $28 \%$. The combination of genes rs $1045642<=2.5$ + rs $12041331>2.5+$ rs662<=2.5 + rs4977574>1.5 was revealed in $26 \%$ and the following combination rs762551<=1.5 + rs13431554<=1.5 + rs291096>1.5 + rs $12248560<=1.5$ in $20 \%$.

The following assumptions were applied when analyzing the results of patients with a fixed stent without restenosis and a fixed stent with restenosis ( $p$ had a higher value, but close to $5 \%$ ). Thus, three SNPs associated with stenting were identified: $r s 7543130(p=0.009324), \quad r s 6785930$ $(p=0.016858)$, and $r s 7819412(p=0.061325)$ and two SNPs associated with the development of restenosis after stent placement: $r s 1061781(p=0.063184)$ and rs342293 ( $p=0.061636)$.

It should be noted that the Bonferroni correction for multiple comparisons did not reveal significant polymorphisms associated with the development of restenosis after stent fixing, which requires further research to increase the sample.

\section{Discussion}

As a result of the analysis of genotyping data, the polymorphisms associated with the risk of developing restenosis after stenting were determined: Codominant inheritance pattern - one polymorphism (rs1045642, $\mathrm{p}=0.0427$ ); dominant inheritance pattern - three polymorphisms ( $r$ 12041331, $p=0.036088$; rs13431554, $p=0.025461 ; r s 1045642, p=0.012774)$, and overdominant inheritance pattern - one polymorphism ( $r$ 13431554, $p=0.036006)$.

Using machine learning, a diagnostic algorithm was obtained for assessing the association of polymorphisms with the risk of developing restenosis after stenting in the form of a decision tree based on the determination of the genotypes of rs 1045642 polymorphism.

Based on the hybrid machine learning approach (RuleFit), four rules were obtained for assessing the empirical risk of restenosis developing after stenting from $20 \%$ to $40 \%$.

The conducted studies allow considering the established polymorphisms as predictors of the repeated stenosis development of the coronary arteries in the stent installation area, which determines the importance of early genetic examination of patients with chronic coronary syndrome to develop the comprehensive treatment tactics and patient monitoring. The proposed decision tree with rs1045642 genotypes allows evaluating the pre-test probability of restenosis developing in patients with chronic coronary syndrome.

\section{Conclusion}

Thus, using machine learning, a diagnostic algorithm was obtained for assessing the association of polymorphisms with the risk of developing restenosis after stenting in the form of a decision tree, which is based on the determination of rs 1045642 polymorphism genotypes. Based on the hybrid approach of machine learning (RuleFit), 4 rules for assessing the empirical risk of developing restenosis after stenting from 20 to $40 \%$ were obtained.

\section{References}

1. Antiplatelet Trialists' Collaboration. Collaborative overview of randomised trials of antiplatelet therapy Prevention of death, myocardial infarction, and stroke by prolonged antiplatelet therapy in various categories of patients. BMJ 1994;308:81-106. 


\section{PMid:8298418}

2. Bhatt DL, Hulot JS, Moliterno DJ, Harrington R. Antiplatelet and anticoagulation therapy for acute coronary syndromes. Circ Res 2014;114:1929-43.

PMid:24902976

3. Hulot JS, Bura A, Villard E, Azizi M, Remones V, Goyenvalle C, et al. Cytochrome P450 2C19 loss of function polymorphism is a major determinant of clopidogrel responsiveness in healthy subjects. Blood 2006;108:2244-7.

PMid:16772608

4. Sardu C. Effects of metformin therapy on coronary endothelial dysfunction in prediabetic patients with stable angina and non-obstructive coronary artery stenosis: The CODYCE multicenter prospective study. Diabetes Care 2019;42(10):1946-55

PMid:30796109

5. Wang L, Wang X, Chen F. Clopidogrel resistance is associated with long-term thrombotic events in patients implanted with drug-eluting stents. Drugs R D. 2010;10(4):219-24. https://doi. org/10.2165/11539580-000000000-00000

\section{PMid:21171668}

6. Gawrońska-Szklarz B. Influence of CYP2C19, ABCB1 and PON1 genotypes on the plasma concentrations of clopidogrel metabolite and platelet aggregation in patients after coronary stenting (PCI). Pharmacol Rep 2013;65:46.

7. Breet NJ, van Werkum JW, Bouman HJ, Kelder JC, Ruven HJ, Bal ET, et al. Comparison of platelet function tests in predicting clinical outcome in patients undergoing coronary stent implantation. JAMA. 2010;303(8):754-62. https://doi. org/10.1001/jama.2010.181

\section{PMid:20179285}

8. Parodi G, Marcucci R, Valenti R, Gori AM, Migliorini A Giusti $B$, et al. High residual platelet reactivity after clopidogrel loading and long-term cardiovascular events among patients with acute coronary syndromes undergoing PCl. JAMA. 2011;306(11):1215-23. https://doi.org/10.1001/jama.2011.1332 PMid:21934054

9. Serebruany VL, Steinhubl SR, Berger PB, Malinin AI, Bhatt DL, Topol EJ. Variability in platelet responsiveness to clopidogrel among 544 individuals. J Am Coll Cardiol. 2005;45(2):246-51. https://doi.org/10.1016/j.jacc.2004.09.067 PMid:15653023

10. Zholdybayeva EV, Talzhanov YA, Aitkulova AM, Tarlykov PV, Kulmambetova $\mathrm{GN}$, Iskakova $\mathrm{AN}$, et al. Genetic risk factors for restenosis after percutaneous coronary intervention in Kazakh population. Hum Genomics. 2016;10(1):15. https://doi. org/10.1073/pnas.1816748116

11. Smagulova G, Kulmurzaeva $\mathrm{N}$, Seytmaganbetova $\mathrm{N}$, Kurmanalina G, Talipova I. The first results of the prevalence of cyp2c19 genepolymorphism in patients with acute coronary syndrome in the Aktyubinsk population. Georgian Med News. 2016;253:61-6. https://doi.org/10.1186/s40246-016-0077-z PMid:27249437

12. Friedman $\mathrm{JH}$, Popescu BE. Predictive learning via rule ensembles. Ann Appl Stat. 2008;2(3):916-54. https://doi. org/10.1214/07-aoas148

13. Luna JM, Gennatas ED, Ungar LH, Eaton E, Diffenderfer ES, Jensen ST, et al. Building more accurate decision trees with the additive tree. Proc Natl Acad Sci U S A. 2019;116(40):19887-93. PMid:31527280 